\title{
Effect of Prophylactic Antifungal Protocols on the Prognosis of Liver Transplantation: A Propensity Score Matching and Multistate Model Approach
}

\author{
Yi-Chan Chen, ${ }^{1,2,3}$ Ting-Shuo Huang, ${ }^{1,4,5}$ Yu-Chao Wang, ${ }^{3}$ \\ Chih-Hsien Cheng, ${ }^{3}$ Chen-Fang Lee, ${ }^{3}$ Ting-Jun Wu, ${ }^{3}$ Hong-Shiue Chou, ${ }^{3}$ \\ Kun-Ming Chan, ${ }^{3}$ Wei-Chen Lee, ${ }^{3}$ and Ruey-Shyang Soong ${ }^{1,2,3}$ \\ ${ }^{1}$ Department of General Surgery, Keelung Chang Gung Memorial Hospital, Keelung 204, Taiwan \\ ${ }^{2}$ School of Medicine, Chang Gung University, Taoyuan 259, Taiwan \\ ${ }^{3}$ Department of Liver and Transplantation Surgery, Linkou Chang Gung Memorial Hospital, Taoyuan 259, Taiwan \\ ${ }^{4}$ Department of Chinese Medicine, College of Medicine, Chang Gung University, Taoyuan 259, Taiwan \\ ${ }^{5}$ Community Medicine Research Center, Keelung Chang Gung Memorial Hospital, Keelung 204, Taiwan \\ Correspondence should be addressed to Ruey-Shyang Soong; kodlp62@gmail.com
}

Received 22 April 2016; Revised 18 August 2016; Accepted 31 August 2016

Academic Editor: Anand Kumar

Copyright (c) 2016 Yi-Chan Chen et al. This is an open access article distributed under the Creative Commons Attribution License, which permits unrestricted use, distribution, and reproduction in any medium, provided the original work is properly cited.

\begin{abstract}
Background. Whether routine antifungal prophylaxis decreases posttransplantation fungal infections in patients receiving orthotopic liver transplantation (OLT) remains unclear. This study aimed to determine the effectiveness of antifungal prophylaxis for patients receiving OLT. Patients and Methods. This is a retrospective analysis of a database at Chang Gung Memorial Hospital. We have been administering routine antibiotic and prophylactic antifungal regimens to recipients with high model for end-stage liver disease scores $(>20)$ since 2009. After propensity score matching, 402 patients were enrolled. We conducted a multistate model to analyze the cumulative hazards, probability of fungal infections, and risk factors. Results. The cumulative hazards and transition probability of "transplantation to fungal infection" were lower in the prophylaxis group. The incidence rate of fungal infection after OLT decreased from $18.9 \%$ to $11.4 \%(p=0.052)$; overall mortality improved from $40.8 \%$ to $23.4 \%(p<0.001)$. In the "transplantation to fungal infection" transition, prophylaxis was significantly associated with reduced hazards for fungal infection (hazard ratio: $0.57,95 \%$ confidence interval: $0.34-0.96, p=0.033$ ). Massive ascites, cadaver transplantation, and older age were significantly associated with higher risks for mortality. Conclusion. Prophylactic antifungal regimens in high-risk recipients might decrease the incidence of posttransplant fungal infections.
\end{abstract}

\section{Introduction}

Orthotopic liver transplantation (OLT) is the treatment of choice for patients with hepatocellular carcinoma, end-stage liver disease, and acute liver failure [1]. Despite advances in surgical techniques, availability of immunosuppressants, and evidence-based guidelines for perioperative management to improve the overall survival of transplant recipients, rejections and infections still affect early posttransplantation mortality. Although advances in immunosuppressants has decreased the incidence of organ rejections, recipients are at greater risk of infections [2]. The use of immunosuppressants has been found to affect host immunity, causing recipients to become susceptible to viral and fungal infections, and subsequently death, after OLT $[2,3]$. In addition, several lines of evidence reveal that intensive care unit conditions, surgical techniques, type of transplantation, type of anastomosis method, massive blood transfusion, and prophylactic antibiotics and immunosuppressants are associated with posttransplant fungal infections [4]. Despite the advances in surgical techniques leading to reductions in intraoperative blood transfusions and surgical time in recent years, the incidence of invasive fungal infection (IFI) still ranges from $5 \%$ to $20 \%[2,5,6]$. 
IFI is the major cause of mortality in the early posttransplantation state. IFI-related mortality in organ transplantation has been found to cause up to $77 \%$ of deaths, one of the major causes of early posttransplantation mortality [6]. Among IFIs, Candida species are the most common pathogens, followed by Aspergillus species. Before 2009, antifungal prophylaxis was controversial owing to the lack of direct evidence that it improved survival. However, a prophylactic antifungal regimen for transplant recipients at high risk of fungal infection was suggested by the evidencebased guidelines of the Infectious Disease Society of America (IDSA) in 2009 [7]. Nevertheless, there is little direct evidence with regard to prognosis after such prophylactic strategies. Thus, we conducted a retrospective hospital-based cohort study to investigate whether routine antifungal prophylaxis regimens reduce the risk of fungal infections in patients receiving OLT. In addition, we conducted a multistate model to investigate transition-specific risk factors.

\section{Patients and Methods}

2.1. Study Cohort. Patients undergoing OLT between January 2005 and September 2014 at the Chang Gung Memorial Hospital, Linkou, were enrolled retrospectively and were followed up until December 2015. All patients receiving either deceased or living donor livers (LDLT) were enrolled, and routine screening of infections following OLT was conducted. Routine culture from ascites and catheter were conducted perioperatively and sputum culture was conducted routinely for patients under mechanical ventilation. Patients with fungal infection before transplantation were excluded to prevent overestimation of the incidence. Clinical data, including age, sex, type of hepatitis, status of liver cirrhosis, model for endstage liver disease (MELD) score, indication for OLT, type of OLT, microbiological screening results, and status of ascites after OLT, were collected. Ethical approval was obtained from the Committee of Ethics in Biomedical Research of Chang Gung Memorial Hospital, and the study conformed to the ethical guidelines of the 1975 Declaration of Helsinki.

\subsection{Prophylaxis Strategy. Our intervention group underwent} routine antifungal prophylaxis. At our institution, since 2009, a prophylactic antifungal regimen was routinely provided to transplant recipients with a high (>20) MELD score before undergoing transplantation. Perioperative prophylaxis consisted of ceftriaxone $(2000 \mathrm{mg} /$ day $)$ in two divided doses plus ampicillin sodium $(1000 \mathrm{mg} / \mathrm{q} 6 \mathrm{~h})$ adjusted by renal function for patients with a lower MELD score $(\leq 20)$ and vancomycin $\mathrm{HCl}(15 \mathrm{mg} / \mathrm{kg} /$ dose q12 h) adjusted by renal function plus Tienam (imipenem (500 mg)/cilastatin (500 mg); $500 \mathrm{mg} / \mathrm{q} 6 \mathrm{~h}$ ) adjusted by renal function for patients with a high MELD score $(>20)$. We used echinocandins, either anidulafungin (100 mg/day) or micafungin (100 mg/day), for fungal prophylaxis to prevent drug interactions between the antifungal agents and the immunosuppressants (calcineurin inhibitors) [8].
2.3. Diagnosis of Fungal Infection. Our primary outcome was risk for fungal infection in patients with liver transplantation. The diagnosis of fungal infection was based on positive culture data after OLT, which revealed a specific fungus or positive findings of yeast in the blood, wound, urine, catheter, or sputum. A positive fungal culture from urine combined with clinical manifestations was identified as a fungal infection instead of colonization. Positive cultures from blood, urine, and sputum depended on the clinical manifestation to define it as an infection.

2.4. Statistical Analysis. Continuous variables were summarized as median with interquartile range, while categorical variables were presented as frequency and percentage. In univariate analysis, baseline characteristics were compared between the intervention group and nonintervention group using the chi-squared test, Fisher's exact test, or Wilcoxon's rank-sum test, as appropriate. To reduce selection and confounding biases, we conducted propensity score matching using the nearest neighbor matching method with a $1: 1$ ratio for the intervention and nonintervention groups [9]. Furthermore, we used a multistate model to model the "transplant to fungal infection transition," "transplant to death transition," and "fungal infection to death transition," occurring as a result of various reasons $[10,11]$. First, death is a competing event with fungal infection occurrence. Second, we could simultaneously model all 3 transitions and estimate the cause-specific cumulative hazards, as well as causespecific transition probability. In addition, we conducted cause-specific Cox models to investigate predictors of the 3 transitions. We performed model selection by Akaike information criterion (AIC) in a stepwise algorithm and substantive knowledge to find the parsimonious models [12]. In addition, we investigated the proportional hazards assumption using the modified Schoenfeld residuals test [13]. All reported confidence intervals (CIs) and tests were two-sided, with a 5\% significance level. All analyses were performed using $\mathrm{R}$ software version 3.3.1 ( $\mathrm{R}$ Foundation for Statistical Computing, Vienna, Austria) with contributed packages "MatchIt" [9], "MASS" [14], “mstate" [10, 11], and "survival" [13].

\section{Results}

A total of 561 patients were enrolled, of which 360 (64.2\%) received the routine prophylactic antifungal regimen and 201 (35.8\%) did not. After propensity score matching, a total of 402 patients were included for further analysis and the variables were comparable between the two groups. The demographic data before and after matching are presented in Table 1. After matching, the rate of fungal infection was $18.9 \%$ before routine prophylaxis and $11.4 \%$ after prophylactic treatment $(p=0.052)$, and the overall mortality rate of the recipients was $40.8 \%$ before the use of routine prophylaxis and $23.4 \%$ after $(p<0.001)$. Hepatitis $\mathrm{B}$ virus infection was dominant in the OLT recipients, followed by hepatitis $\mathrm{C}$ virus infection. The median time of fungal infection in the prophylaxis group was 27 days (interquartile range (IQR) 10.5-77.7 days), whereas it was 
TABLE 1: Demographic data of the liver transplantation recipients (before and after matching).

\begin{tabular}{|c|c|c|c|c|c|c|}
\hline & \multicolumn{3}{|c|}{ Before matching } & \multicolumn{3}{|c|}{ After matching } \\
\hline & No prophylaxis & Prophylaxis & $p$ value & No prophylaxis & Prophylaxis & $p$ value \\
\hline$N$ & 201 & 360 & & 201 & 201 & \\
\hline Age (median (IQR)) & $53.00(47.00,57.00)$ & $55.00(48.00,60.00)$ & 0.021 & $53.00(47.00,57.00)$ & $54.00(48.00,59.00)$ & 0.228 \\
\hline \multicolumn{7}{|l|}{ Age (\%) } \\
\hline$\leq 55$ & $71(35.3)$ & $123(34.2)$ & \multirow{3}{*}{0.048} & $71(35.3)$ & $69(34.3)$ & \multirow{3}{*}{0.953} \\
\hline$>55-60$ & $101(50.2)$ & $155(43.1)$ & & $101(50.2)$ & $101(50.2)$ & \\
\hline$>60$ & $29(14.4)$ & $82(22.8)$ & & $29(14.4)$ & $31(15.4)$ & \\
\hline \multicolumn{7}{|l|}{ Sex $(\%)$} \\
\hline Male & $153(76.1)$ & $264(73.3)$ & \multirow{2}{*}{0.533} & $153(76.1)$ & $160(79.6)$ & \multirow{2}{*}{0.471} \\
\hline Female & $48(23.9)$ & $96(26.7)$ & & $48(23.9)$ & $41(20.4)$ & \\
\hline \multicolumn{7}{|l|}{ HCC (\%) } \\
\hline No & $112(55.7)$ & $195(54.2)$ & \multirow{2}{*}{0.79} & $112(55.7)$ & $108(53.7)$ & \multirow{2}{*}{0.764} \\
\hline Yes & $89(44.3)$ & $165(45.8)$ & & $89(44.3)$ & $93(46.3)$ & \\
\hline \multicolumn{7}{|l|}{ Viral hepatitis (\%) } \\
\hline None & $26(12.9)$ & $78(21.7)$ & \multirow{4}{*}{0.017} & $26(12.9)$ & $29(14.4)$ & \multirow{4}{*}{0.905} \\
\hline $\mathrm{HBV}$ & $126(62.7)$ & $183(50.8)$ & & $126(62.7)$ & $127(63.2)$ & \\
\hline $\mathrm{HCV}$ & 38 (18.9) & $84(23.3)$ & & 38 (18.9) & $33(16.4)$ & \\
\hline $\mathrm{HBV}+\mathrm{HCV}$ & $11(5.5)$ & $15(4.2)$ & & $11(5.5)$ & $12(6.0)$ & \\
\hline \multicolumn{7}{|l|}{ Ascites (\%) } \\
\hline Mild/moderate $(\leqq 2000 \mathrm{~mL})$ & $139(69.2)$ & $256(71.2)$ & \multirow{2}{*}{0.484} & $139(69.2)$ & $138(68.7)$ & \multirow{2}{*}{1} \\
\hline Massive (>2000 mL) & $62(30.8)$ & $104(28.9)$ & & $62(30.8)$ & $63(31.3)$ & \\
\hline \multicolumn{7}{|l|}{ Living donor (\%) } \\
\hline No & $64(31.8)$ & $72(20.0)$ & \multirow{2}{*}{0.002} & $64(31.8)$ & $58(28.9)$ & \multirow{2}{*}{0.588} \\
\hline Yes & $137(68.2)$ & $288(80.0)$ & & $137(68.2)$ & $143(71.1)$ & \\
\hline \multicolumn{7}{|l|}{ MELD score } \\
\hline$\leqq 20$ & $130(64.7)$ & $258(71.7)$ & \multirow{2}{*}{0.104} & $130(64.7)$ & $133(66.2)$ & \multirow{2}{*}{0.834} \\
\hline$>20$ & $71(35.3)$ & $102(28.3)$ & & $71(35.3)$ & $68(33.8)$ & \\
\hline \multicolumn{7}{|l|}{ Fungal infection (\%) } \\
\hline No & $163(81.1)$ & $315(87.5)$ & \multirow{2}{*}{0.054} & $163(81.1)$ & $178(88.6)$ & \multirow{2}{*}{0.052} \\
\hline Yes & $38(18.9)$ & $45(12.5)$ & & $38(18.9)$ & $23(11.4)$ & \\
\hline \multicolumn{7}{|l|}{ Mortality (\%) } \\
\hline No & $119(59.2)$ & $272(75.6)$ & \multirow{2}{*}{$<0.001$} & $119(59.2)$ & $154(76.6)$ & $<0.001$ \\
\hline Yes & $82(40.8)$ & $88(24.4)$ & & $82(40.8)$ & $47(23.4)$ & \\
\hline Propensity score (median (IQR)) & NA & NA & & $0.40(0.32,0.46)$ & $0.40(0.32,0.44)$ & 0.690 \\
\hline
\end{tabular}

IQR: interquartile range, HCC: hepatocellular carcinoma, and MELD: model for end-stage liver disease. Ascites was measured during the operation.

21 days (IQR 10-48.5 days) in the nonprophylaxis group. The transition matrix of the 3 states is summarized in the Supplemental Table 1 (see Supplementary Material available online at http://dx.doi.org/10.1155/2016/6212503). 61 of 402 (15\%) patients developed "transplantation to fungal infection" transition. Among 61 patients with fungal infection, 36 (59\%) patients died.

The species causing fungal infection are shown in Table 2. The most common fungal infection was by Candida albicans: $33.7 \%$ of infections before routine prophylaxis and 35.9\% after. Aspergillus infection disappeared after initiating the routine prophylactic antifungal regimen. The incidence of Candida glabrata and Candida tropicalis increased after frequent echinocandin usage. Yeast was found in 32.3\% and $28.2 \%$ of the cultures before and after prophylaxis, respectively. Eleven patients in the infected group developed 2 kinds of fungal infections and 1 developed 3 kinds of fungal infections.

A multistate model was used to evaluate the cumulative hazards and transition probability after OLT for "transplantation to fungal infection," "transplantation to death," and "fungal infection to death" transitions. Figure 1 shows that the cumulative hazards of a "transplantation to fungal infection" transition were lower in the routine prophylaxis group compared to the nonprophylaxis group. Cumulative hazards for "transplantation to death" and "fungal infection to death" transitions were similar in the 2 groups. We estimated 1year, 2-year, and 3-year transition probabilities among the four states including "transplantation," "fungal infection," "death with fungal infection," and "death without fungal 
TABLE 2: Species of fungus before and after the prophylactic anti-fungal protocol.

\begin{tabular}{|c|c|c|c|c|c|}
\hline \multicolumn{3}{|c|}{ No prophylactic period } & \multicolumn{3}{|c|}{ Prophylactic period } \\
\hline Species & Number & $\%$ & Species & Number & $\%$ \\
\hline Candida albicans & 25 & 33.7 & Candida albicans & 28 & 35.9 \\
\hline Candida glabrata & 7 & 9.9 & Candida tropicalis & 12 & 15.3 \\
\hline Candida tropicalis & 5 & 6.7 & Candida glabrata & 5 & 6.4 \\
\hline Candida parapsilosis & 4 & 5.4 & Candida parapsilosis & 3 & 3.8 \\
\hline Candida krusei & 2 & 2.7 & Candida krusei & 1 & 1.3 \\
\hline Aspergillus & 3 & 4.0 & Candida guilliermondii & 1 & 1.3 \\
\hline Mold & 2 & 2.7 & Candida sp. & 1 & 1.3 \\
\hline Penicillium sp. & 1 & 1.3 & Mucor sp. & 1 & 1.3 \\
\hline Trichosporon sp. & 1 & 1.3 & Mold & 4 & 5.0 \\
\hline Yeast & 23 & 32.3 & Yeast & 22 & 28.2 \\
\hline
\end{tabular}

Note. Eleven patients developed 2 kinds of fungal infection and 1 developed 3 kinds of fungal infection. Mold and yeast species are not routinely identified without physician's requests.

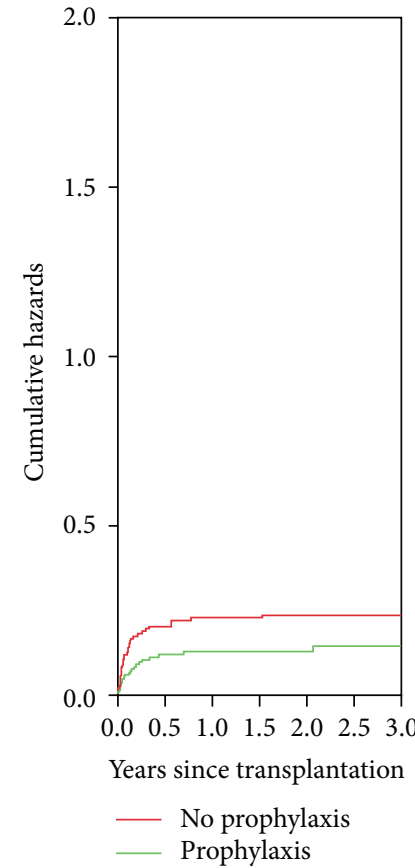

(a) Transplant to fungus

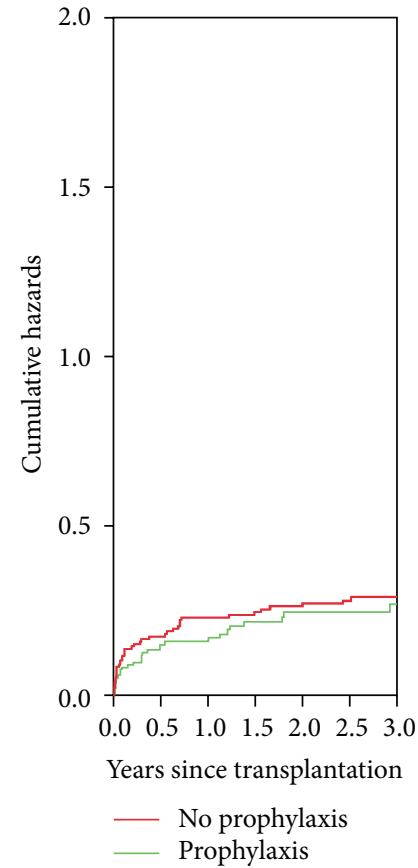

(b) Transplant to death

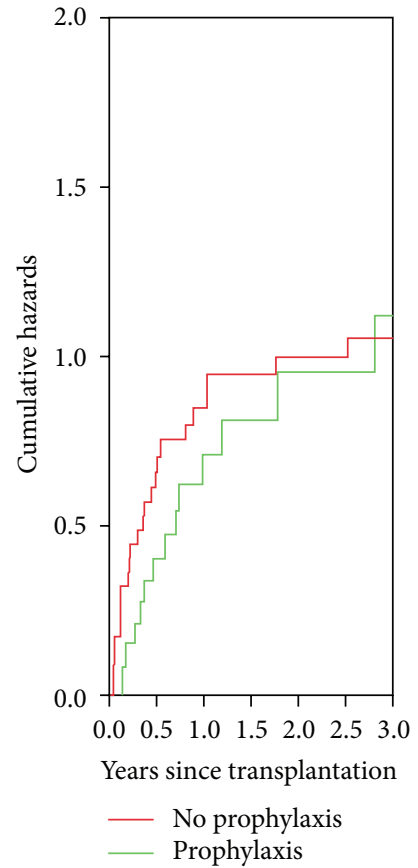

(c) Fungus to death

Figure 1: Nonparametric estimates of cumulative hazards of the multistate model stratified by transitions. (a) Cumulative hazards for the transition from liver transplantation to fungal infection demonstrate the fungal infection which occurred within the early period of transplantation and reached a plateau after 3 months. The routine prophylaxis group has lower cumulative hazards of "transplantation to fungal infection" transition. (b) Cumulative hazards for the transition from liver transplantation to death between the two groups were similar. (c) Cumulative hazards for the transition from fungal infection to death between the two groups were similar.

infection" (Table 3). The routine prophylaxis group had a lower probability of "fungal infection" and "death with fungal infection." Notably, the sum of "fungal infection" and "death with fungal infection" probability did not obviously increase over time, indicating most fungal infections occurred in the early posttransplantation period.

We also investigated predictors in 3 transition-specific multivariable Cox models (Table 4). In the transition from "transplantation to fungal infection," the routine prophylaxis group was significantly associated with reduced hazards for fungal infection compared with the nonprophylaxis group (hazard ratio (HR): $0.57,95 \%$ confidence interval (CI): $0.34-$ 0.96, $p=0.033)$. In the transition from "transplantation to death," patients with massive ascites were associated with a higher risk for mortality compared to patients with mild/moderate ascites (HR: 1.55, 95\% CI: 1.02-2.36, $p=$ 0.042). By checking the proportional hazards assumptions, LDLT was statistically significant associated with timevarying effects. Thus, we used the time point of 1.5 years after liver transplantation to model the LDLT effects (this 
TABLE 3: The 1-year, 2-year, and 3-year transition probability among four states in the multistate model.

\begin{tabular}{lccc}
\hline & \multicolumn{2}{c}{ State occupied probability $(95 \%$ CI $)$} \\
2-year
\end{tabular}

TABLE 4: Results of multivariable transition-specific Cox models.

\begin{tabular}{|c|c|c|c|}
\hline Variable & Category & $\mathrm{HR}(95 \% \mathrm{CI})$ & $p$ value \\
\hline \multicolumn{4}{|c|}{ Transition: transplant to fungal infection } \\
\hline \multirow{2}{*}{ Treatment } & No prophylaxis & 1 & \\
\hline & Prophylaxis & $0.57(0.34-0.96)$ & $0.033^{*}$ \\
\hline \multirow{2}{*}{ Ascites } & Mild/moderate & 1 & \\
\hline & Massive & $1.65(0.98-2.76)$ & 0.058 \\
\hline Propensity score & & $0.15(0.01-1.76)$ & 0.132 \\
\hline \multicolumn{4}{|l|}{ Transition: transplant to death } \\
\hline \multirow{2}{*}{ Ascites } & Mild/moderate & 1 & \\
\hline & Massive & $1.55(1.02-2.36)$ & $0.042^{*}$ \\
\hline \multirow{2}{*}{ Living donor (within 1.5 years) } & No & 1 & \\
\hline & Yes & $0.41(0.26-0.66)$ & $<0.001^{*}$ \\
\hline \multirow{2}{*}{ Living donor (after 1.5 years) } & No & 1 & \\
\hline & Yes & $1.08(0.45-2.62)$ & 0.861 \\
\hline \multicolumn{4}{|l|}{ Transition: fungal infection to death } \\
\hline \multirow{3}{*}{ Age (years) } & $\leq 50$ & 1 & \\
\hline & $>50, \leq 60$ & $2.55(1.10-5.93)$ & 0.029 \\
\hline & $>60$ & $1.80(0.67-4.83)$ & 0.240 \\
\hline \multirow{2}{*}{ Ascites } & Mild/moderate & 1 & \\
\hline & Massive & $2.19(1.06-4.52)$ & 0.035 \\
\hline \multirow{2}{*}{ Living donor } & No & 1 & \\
\hline & Yes & $0.57(0.28-1.14)$ & 0.113 \\
\hline
\end{tabular}

HR: hazard ratio and CI: confidence interval. ${ }^{*} p<0.05$.

time point was indicated by the residual plots). LDLT was associated with a lower risk for short-term mortality (with 1.5 years) compared to patients receiving cadaver transplantation (HR: 0.41, CI: $0.26-0.66, p \leq 0.001)$. However, 1.5 years after liver transplantation, LDLT was not significantly associated with a lower risk for mortality. In the transition from "fungal infection to death," older age and massive ascites were significantly associated with a higher risk of mortality.

\section{Discussion}

Our current study demonstrated that routine prophylactic antifungal regimens are associated with a lower risk for "transplantation to fungal infection" in patients receiving
OLT. Patients with massive ascites had a higher risk for "transplantation to death" and "fungal infection to death" transitions. Patients receiving LDLT had a lower risk for the "transplantation to death" transition within 1.5 years after OLT.

Because of the evolution of surgical techniques and improvements in post-OLT management, the 5-year survival rate after OLT has reached $72-77 \%$ in recent times [15]. However, IFI is still one of the major causes of early mortality in liver transplant recipients. The incidence of IFI ranges from $5 \%$ to $20 \%[2,5,6]$. According to the literature, Candida and Aspergillus are the most common causal agents and are associated with high mortality in organ transplantation, accounting for $30-60 \%$ of the infections $[5,16]$. 
The reported risk factors for IFI include retransplantation, dialysis, prolonged operation time, and prolonged broad-spectrum antibiotics use [2, 17]. Fungal infections most frequently occur in the first month after OLT, and antifungal prophylaxis could significantly reduce fungal infections in patients receiving OLT. In a recent metaanalysis, antifungal prophylaxis has been shown to reduce fungal infection-related mortality [18]. Among antifungal drugs, echinocandins, such as caspofungin, micafungin, and anidulafungin, have excellent in vitro activity against Candida species, with few side effects and minimal drug-drug interactions; in particular, they do not influence the clearance of calcineurin inhibitors, which are commonly used immunosuppressants. Moreover, dosage adjustment is not required in patients with impaired renal function or those under dialysis [19].

Although advances in perioperative management and surgical techniques enable improved survival of OLT recipients, an infection after OLT is a major cause of mortality. A prophylactic protocol was established at our institution since 2009, which includes empiric antibiotics based on the MELD score and a regimen of prophylactic antifungal treatment in high-risk patients. IDSA guidelines have suggested prophylactic antifungal treatment in patients with renal dysfunction, retransplantation, or reoperation; however, more recent guidelines suggest prophylaxis in high-risk patients with high MELD scores, choledochojejunostomy, bile leaks, and LDLT [17, 20, 21]. Our current study showed that routine antifungal regimens reduce the risk for a "transplantation to fungal infection" transition. In addition, our results suggest fungal infections mostly occurred within the initial 3 months after OLT, which is consistent with other reports.

Most infections, either bacterial or fungal, occur in the first month after OLT, causing early mortality after transplantation in the first year [22]. In the current study, 1-year, 2 -year, and 3-year probability of fungal infection and death with fungal infection were reduced in the prophylaxis group. These results indicate prophylactic protocols might reduce the incidence of fungal infection and death with fungal infection. The IDSA guideline in 2009 recommended routine antifungal prophylaxis for OLT recipients [23]. Recently, Saliba et al. reported that a MELD score of $>30$ might be the most important risk factor for IFI [17]. Patients with a MELD score $>20$ have a higher possibility of pretransplantation renal dysfunction and liver dysfunction; therefore, we use this threshold for prophylaxis.

Patients undergoing OLT can have several timedependent outcomes during follow-up [24]. A multistate model has been applied to analyze competing risks in patients with liver cirrhosis [25]. By evaluating the transition-specific risk factors from the multistate model, we found that a routine prophylactic antifungal regimen in high-risk recipients prevents further fungal infection. In patients with fungal infections, C. albicans was the most common species before and after prophylaxis. The incidence of other species was reduced after prophylaxis. Such findings are consistent with the literature [26]. Intrinsic resistance or resistance induced by the prophylactic agent might account for these findings. In addition, acquired resistance to echinocandins has been reported even in clinically relevant Candida spp. [27]. Acquired resistance species are associated with high mortality after fungal infections. Changing antifungal treatment from echinocandins to azoles or to liposomal amphotericin B should be considered if a positive fungal culture persists even after the antifungal regimen treatment. However, more data need to be collected to confirm this approach. In our institution, the initial positive culture of fungal infection revealed only yeast or molds, and further differentiation of the species required special cultures. If the patients' general condition improved after treatment or the condition became worse, we may not perform specific cultures for further differentiation and that is why almost one-third of the cultures revealed yeast only.

LDLT is associated with a lower short-term risk of "transplant to death" transition. The time-dependent effects might be associated with high-risk patients leaving the riskset in the early period. LDLT might be associated with a shorter waiting time for the organ and prevention of the deterioration of liver function in the recipients. In addition, more reserve liver function and improved surgical techniques may improve long-term survival. Additionally, massive ascites indicates decompensated liver function and leads to about $50 \%$ mortality 2 years after patients present with uncontrolled ascites [28].

Our study has several advantages. First, we used propensity score matching to reduce selection and confounding biases in this observational study. Second, we used time to event outcomes in the current study. Such an approach allowed us to investigate time-varying treatment effects and adjust for competing risks (mortality is a competing risk for fungal infections, because it prevents the occurrence of fungal infection). If we did not adjust for competing risks, we would overestimate the cumulative incidence of fungal infection. Third, we used a multistate model to investigate the transition-specific risk factors.

Nevertheless, our present study has several limitations. First, unmeasured confounders could not be matched. For example, we could not match important biological data and donor-related factors. Second, time-lag bias can compromise the results in such a long-term observational study. For example, the reduced risk of fungal infection might be associated with more experience in surgical technique, improved intensive unit care, and better surgical facilities. Third, the small sample size in the current study limits us from thoroughly investigating predicting factors. Finally, this retrospective analysis of observational hospital-based cohort data might have information and performance biases.

\section{Conclusion}

We conclude that administering routine empiric antibiotic treatment and a prophylactic antifungal regimen to high-risk patients might reduce the incidence of fungal infection in the early stage after liver transplantation and prevent fungal infection-related mortality, which might lead to better longterm survival. Candida species remain the major cause of fungal infection despite prophylaxis. Further clinical trials are warranted to confirm our results. 


\section{Disclosure}

This study is based in part on data from the database of liver transplantation in Chang Gung Memorial Hospital. The interpretations and conclusions contained herein do not represent those of the Chang Gung Memorial Hospital.

\section{Competing Interests}

The authors have no competing interests to declare.

\section{Acknowledgments}

This study was sponsored by the Chang Gung Memorial Hospital Research Projects (CMRPG2D0422, CMRPG2D0832, and CMRPG2C0372).

\section{References}

[1] "National Institutes of Health Consensus Development Conference Statement: liver transplantation-June 20-23, 1983," Hepatology, vol. 4, no. 1, supplement, pp. 107S-110S, 1984.

[2] M. J. Pacholczyk, B. Lagiewska, W. Lisik, D. Wasiak, and A. Chmura, "Invasive fungal infections following liver transplantation-risk factors, incidence and outcome," Annals of Transplantation, vol. 16, no. 3, pp. 14-16, 2011.

[3] C.-H. Yang, X.-S. He, J. Chen et al., "Fungal infection in patients after liver transplantation in years 2003 to 2012," Annals of Transplantation, vol. 17, no. 4, pp. 59-63, 2012.

[4] L. A. Collins, M. H. Samore, M. S. Roberts et al., "Risk factors for invasive fungal infections complicating orthotopic liver transplantation," Journal of Infectious Diseases, vol. 170, no. 3, pp. 644-652, 1994.

[5] P. G. Pappas, B. D. Alexander, D. R. Andes et al., "Invasive fungal infections among organ transplant recipients: results of the transplant-associated infection surveillance network (TRANSNET)," Clinical Infectious Diseases, vol. 50, no. 8, pp. 1101-1111, 2010.

[6] N. Singh, "Fungal infections in the recipients of solid organ transplantation," Infectious Disease Clinics of North America, vol. 17, no. 1, pp. 113-134, 2003.

[7] P. G. Pappas and F. P. Silveira, "Candida in solid organ transplant recipients," American Journal of Transplantation, vol. 9, supplement 4, pp. S173-S179, 2009.

[8] D. W. Denning, "Echinocandin antifungal drugs," The Lancet, vol. 362, no. 9390, pp. 1142-1151, 2003.

[9] D. E. Ho, K. Imai, G. King, and E. A. Stuart, "MatchIt: nonparametric preprocessing for parametric causal inference," Journal of Statistical Software, vol. 42, no. 8, pp. 1-28, 2011.

[10] H. Putter, M. Fiocco, and R. B. Geskus, "Tutorial in biostatistics: competing risks and multi-state models," Statistics in Medicine, vol. 26, no. 11, pp. 2389-2430, 2007.

[11] L. C. de Wreede, M. Fiocco, and H. Putter, "mstate: an R package for the analysis of competing risks and multi-state models," Journal of Statistical Software, vol. 38, no. 7, pp. 1-30, 2011.

[12] F. Kemp, "Modern applied statistics with S," Journal of the Royal Statistical Society: Series D (The Statistician), vol. 52, no. 4, pp. 704-705, 2003.

[13] T. M. Therneau and P. M. Grambsch, Modeling Survival Data: Extending the Cox Model, Statistics for Biology and Health, Springer Science \& Business Media, 2000.
[14] W. N. Venables and B. D. Ripley, Modern Applied Statistics with S, Springer, New York, NY, USA, 4th edition, 2002.

[15] R. M. Merion, "Current status and future of liver transplantation," Seminars in Liver Disease, vol. 30, no. 4, pp. 411-421, 2010.

[16] P. Muñoz, C. Rodríguez, E. Bouza et al., "Risk factors of invasive aspergillosis after heart transplantation: protective role of oral itraconazole prophylaxis," American Journal of Transplantation, vol. 4, no. 4, pp. 636-643, 2004.

[17] F. Saliba, V. Delvart, P. Ichaï et al., "Fungal infections after liver transplantation: outcomes and risk factors revisited in the MELD era," Clinical Transplantation, vol. 27, no. 4, pp. E454E461, 2013.

[18] M. Cruciani, C. Mengoli, M. Malena, O. Bosco, G. Serpelloni, and P. Grossi, "Antifungal prophylaxis in liver transplant patients: a systematic review and meta-analysis," Liver Transplantation, vol. 12, no. 5, pp. 850-858, 2006.

[19] C. A. Kauffman and P. L. Carver, "Update on echinocandin antifungals," Seminars in Respiratory and Critical Care Medicine, vol. 29, no. 2, pp. 211-219, 2008.

[20] F. P. Silveira and S. Kusne, "Candida infections in solid organ transplantation," American Journal of Transplantation, vol. 13, supplement 4, pp. 220-227, 2013.

[21] J. Fortún, P. Martín-Dávila, S. Moreno et al., "Risk factors for invasive aspergillosis in liver transplant recipients," Liver Transplantation, vol. 8, no. 11, pp. 1065-1070, 2002.

[22] F. A. Romero and R. R. Razonable, "Infections in liver transplant recipients," World Journal of Hepatology, vol. 3, no. 4, pp. 83-92, 2011.

[23] P. G. Pappas, C. A. Kauffman, D. Andes et al., "Clinical practice guidelines for the management of candidiasis: 2009 update by the Infectious Diseases Society of America," Clinical Infectious Diseases, vol. 48, no. 5, pp. 503-535, 2009.

[24] P. K. Andersen, R. B. Geskus, T. de witte, and H. Putter, "Competing risks in epidemiology: possibilities and pitfalls," International Journal of Epidemiology, vol. 41, no. 3, pp. 861-870, 2012.

[25] P. Jepsen, H. Vilstrup, and P. K. Andersen, "The clinical course of cirrhosis: the importance of multistate models and competing risks analysis," Hepatology, vol. 62, no. 1, pp. 292-302, 2015.

[26] B. J. Kullberg and M. C. Arendrup, "Invasive candidiasis," The New England Journal of Medicine, vol. 373, no. 15, pp. 1445-1456, 2015.

[27] M. C. Arendrup and D. S. Perlin, "Echinocandin resistance: an emerging clinical problem?" Current Opinion in Infectious Diseases, vol. 27, no. 6, pp. 484-492, 2014.

[28] P. Ginés, E. Quintero, V. Arroyo et al., "Compensated cirrhosis: natural history and prognostic factors," Hepatology, vol. 7, no. 1, pp. 122-128, 1987. 


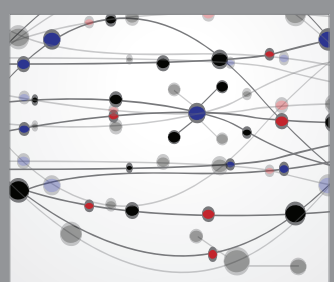

The Scientific World Journal
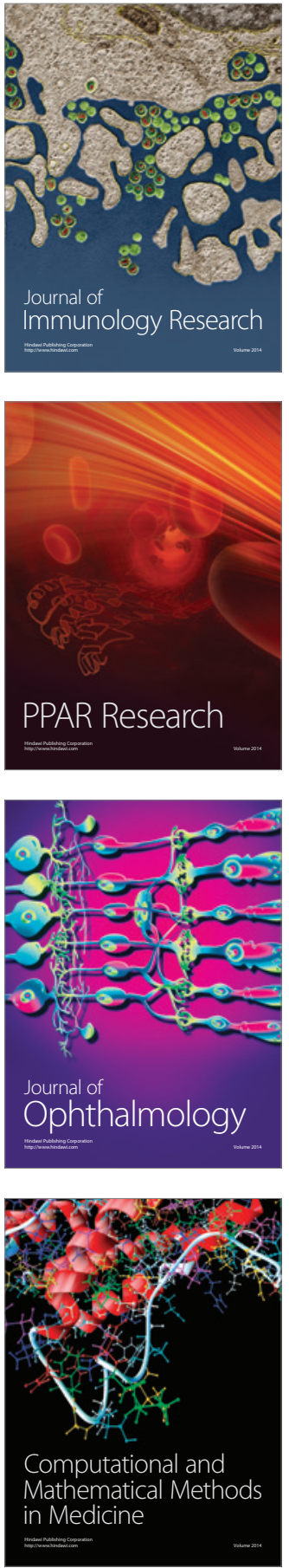

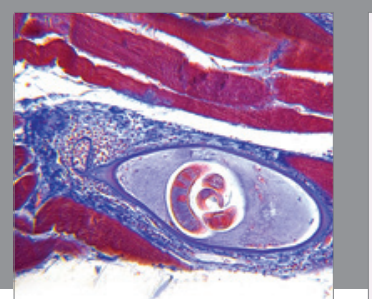

Gastroenterology Research and Practice

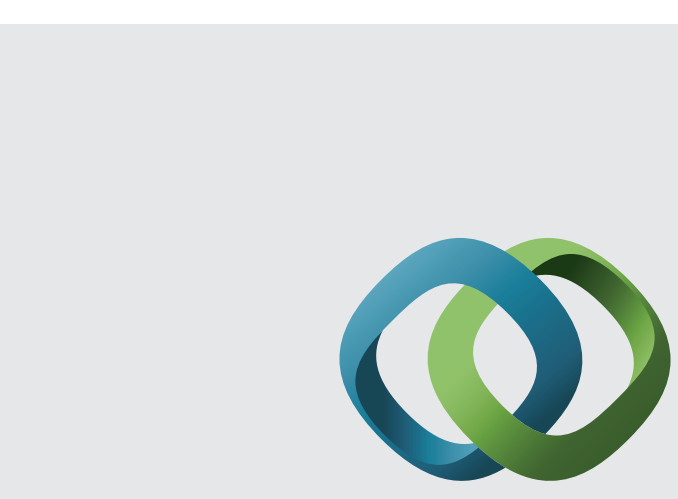

\section{Hindawi}

Submit your manuscripts at

http://www.hindawi.com
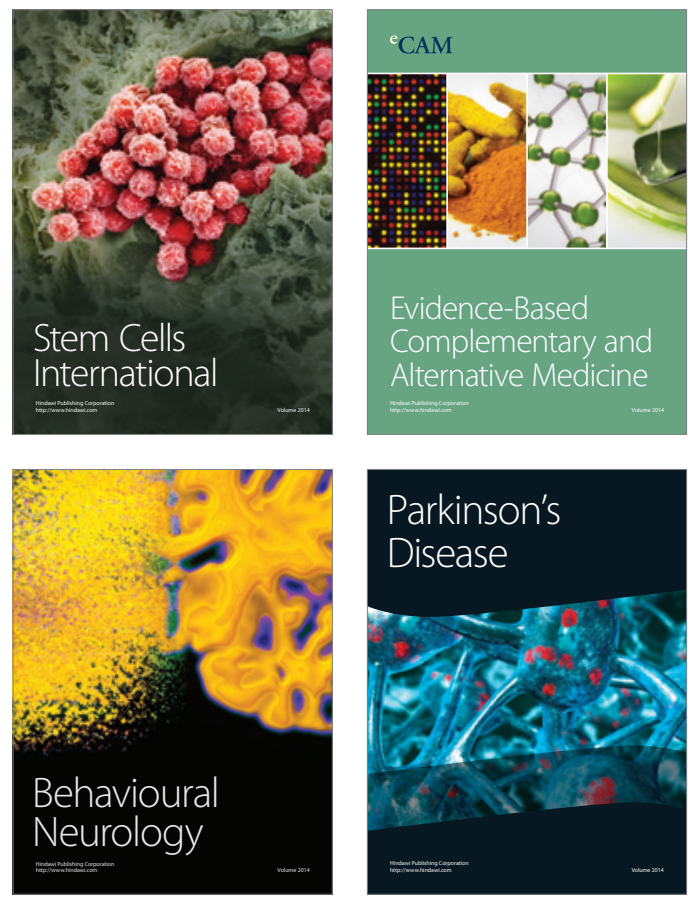
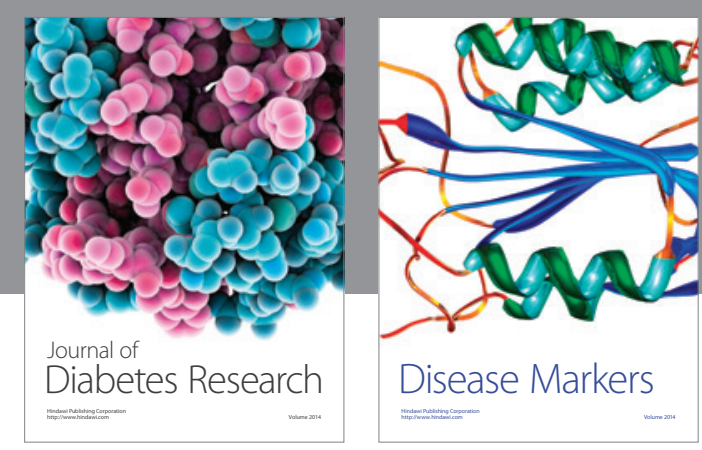

Disease Markers
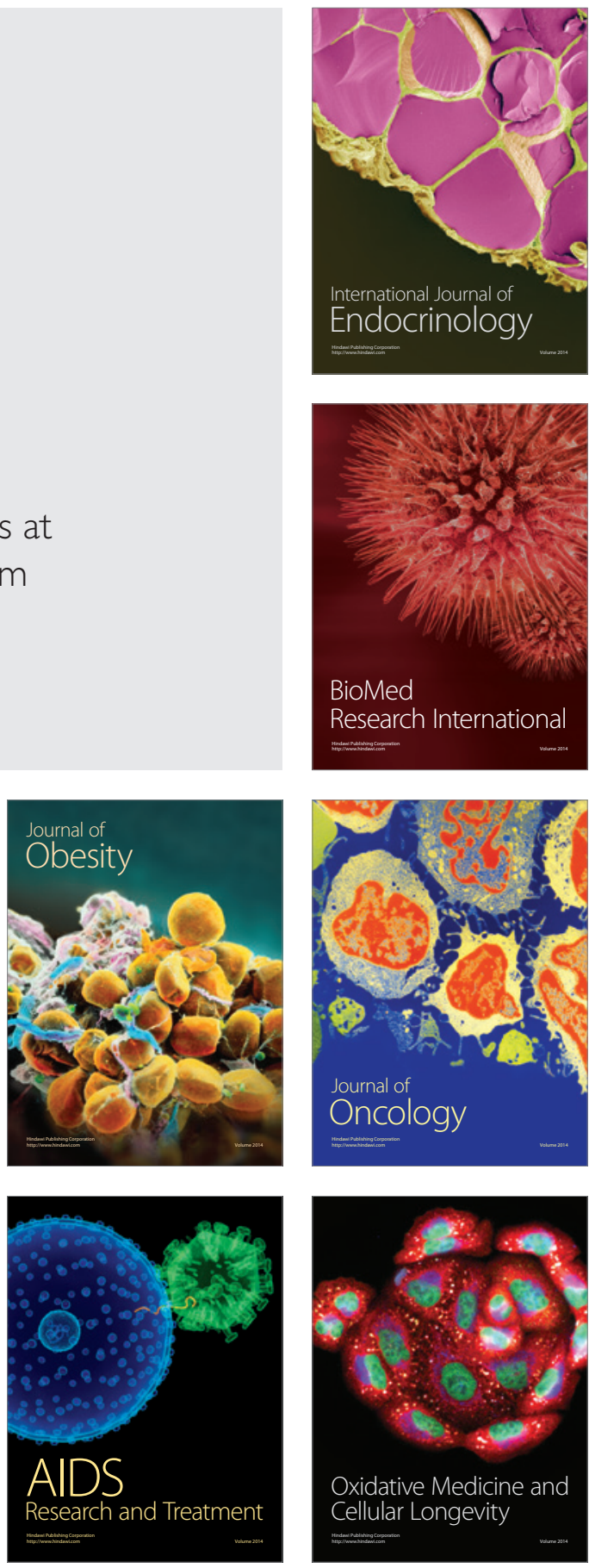